\title{
Lesiones gravísimas causadas por la actuación ilegítima y el terrorismo de Estado en Uruguay. Reporte de diez años de valoración médico-legal y psiquiátrica a las víctimas
}

\author{
Victoria Iglesias*, Valeria da Trindade`, Hugo Rodríguez Almada ${ }^{\ddagger}$
}

\section{Resumen}

El rol de la Medicina Legal en derechos humanos y acción forense humanitaria es cada vez más visible. La ley № 18.596 indemiza a quienes acrediten haber sufrido lesiones gravísimas por el accionar de agentes del Estado.

Objetivo: contribuir al conocimiento público de la actuación ilegítima y el terrorismo de Estado en Uruguay (13/6/1968 y 28/2/1985) y del aporte de la Medicina Legal en materia de verdad, justicia, memoria, reparación y garantía de no repetición. Material y método: se estudiaron las solicitudes de indemnización por lesiones gravísimas recibidas en los primeros diez años de vigencia de la ley.

Resultados y discusión: de 166 peticiones, se admitieron 115. En el 95\% fue requerido informe médico-legal o psiquiátrico, 0 ambos. Aunque fue dificultoso demostrar el nexo causal, en 55 casos se estableció un nexo con razonable grado de certeza. La edad media de las víctimas al momento de producirse las lesiones era de 26 años. La edad media al momento de la indemnización fue de 66 años. Las lesiones demostradas se corresponden con la referida en la bibliografía nacional y extranjera. Se destaca el hallazgo de una considerable presencia de trastornos psicóticos.

Conclusiones: se indemnizaron 115 víctimas por lesiones gravísimas causadas en su amplia mayoría por prisión política prolongada y tortura. Se observó alta prevalencia de patologías osteoarticulares, trastornos por estrés postraumático crónico y depresión, pero también un alto número de psicosis crónicas. Los informes médico-legales fueron relevantes para demostrar las lesiones gravísimas y su nexo causal con el accionar de los agentes del Estado.

Palabras clave: Terrorismo

Heridas y traumatismos

Tortura

Violaciones de los derechos humanos

Medicina legal

Uruguay
Key words: Terrorism

Wounds and injuries

Torture

Human rights abuses

Forensic medicine

Uruguay

\footnotetext{
* Asistente del Departamento de Medicina Legal y Ciencias Forenses de la Facultad de Medicina de la Universidad de la República. Médica del Mecanismo Nacional de Prevención de la Tortura. Institución Nacional de Derechos Humanos y Defensoría del Pueblo.

† Doctora en Derecho. Asesora letrada de la Comisión Especial creada por la ley № 18.596.

‡ Profesor director del Departamento de Medicina Legal y Ciencias Forenses de la Facultad de Medicina de la Universidad de la República.

Departamento de Medicina Legal y Ciencias Forenses. Facultad de Medicina - Universidad de la República

Correspondencia: Dr. Hugo Rodríguez.Correo electrónico: hrodriguez@fmed.edu.uy

Recibido: 4/4/2020

Aprobado: $29 / 6 / 2020$
} 


\section{Introducción}

A nivel mundial es cada vez más visible el rol de la Medicina Legal en materia de derechos humanos y de acción forense humanitaria, sea como contribución a las investigaciones judiciales o bajo otras modalidades que permitan lograr los objetivos de verdad, justicia, memoria, reparación de las víctimas y garantía de no repetición ${ }^{(1-6)}$.

En Uruguay, la investigación de las violaciones a los derechos humanos en el pasado reciente ha contado con una fuerte contribución de la Medicina Legal. El Departamento de Medicina Legal y Ciencias Forenses de la Facultad de Medicina de la Universidad de la República ha producido diversos informes y dictámenes a solicitud de familiares de víctimas, de la Comisión para la Paz, de la Comisión Especial de la ley No 18.596 , de diversas sedes judiciales de varios departamentos y de la Fiscalía Especializada en Crímenes de Lesa Humanidad ${ }^{(7-11)}$.

La ley $\mathrm{N}^{\circ} 18.596$ (Reconocimiento y reparación a las víctimas) de 18 de setiembre de 2009 reconoció "el quebrantamiento del Estado de Derecho que impidiera el ejercicio de los derechos fundamentales de las personas, en violación a los Derechos Humanos o a las normas del Derecho Internacional Humanitario, en el periodo comprendido desde el 27 de junio de 1973 hasta el 28 de febrero de 1985" (Art. $1^{\circ}$ ) y "la responsabilidad del Estado uruguayo en la realizacioón de prácticas sistemáticas de tortura, desaparición forzada y prisión sin intervención del Poder Judicial, homicidios, aniquilación de personas en su integridad psicofisica, exilio político o destierro de la vida social, en el periodo comprendido desde el 13 de junio de 1968 hasta el 26 de junio de 1973" (Art. $2^{\circ}$ ), así como el "derecho a la reparación integral a todas aquellas personas que, por acción $u$ omisión del Estado, se encuentren comprendidas en las definiciones de los artículos $4^{\circ}$ y $5^{\circ}$ de la presente ley" (Art. $3^{\circ}$ ), a saber:

a) Víctimas del terrorismo de Estado: personas que hayan sufrido la violación a su derecho a la vida, a su integridad psicofísica y a su libertad dentro y fuera del territorio nacional entre 27 de junio de 1973 y 28 de febrero de 1985, por motivos políticos, ideológicos o gremiales (Art. $\left.4^{\circ}\right)$.

b) Víctimas de la actuación ilegítima del Estado: personas que hayan sufrido violación a su derecho a la vida, a su integridad psicofísica o a su libertad sin intervención del Poder Judicial dentro o fuera del territorio nacional, desde 13 de junio de 1968 hasta 26 de junio de 1973, por motivos políticos, ideológicos o gremiales (Art. $5^{\circ}$ ).

En ambos casos la norma establece que "las violaciones deberán haber sido cometidas por parte de agen- tes del Estado o de quienes, sin serlo, hubiesen contado con la autorización, apoyo o su aquiescencia" (Arts. $4^{\circ}$ y $5^{\circ}$ ).

La resolución sobre las solicitudes de amparo están a cargo de una Comisión Especial integrada por un delegado del Ministerio de Educación y Cultura, que la preside, uno del Ministerio de Economía y Finanzas, uno del Ministerio de Salud Pública y dos delegados designados por el Poder Ejecutivo a propuesta de las organizaciones representativas de las víctimas del terrorismo de Estado que decide por mayoría absoluta de integrantes (Arts. $16^{\circ}$ y $\left.17^{\circ}\right)$.

Entre las diversas modalidades reparatorias (pecuniarias, sanitarias o simbólicas) previstas en la norma, se cuenta la que asigna una idemnización monetaria por única vez a las "víctimas que hubiesen sufrido lesiones gravisimas a raíz o en ocasión del accionar de agentes del Estado" (Art. 11º, Lit. B). Para la toma de decisiones en las solicitudes de amparo por esta causal, la Comisión Especial recurre al asesoramiento de médicos legistas o psiquiatras, o ambos, que dictaminan sobre las dos condiciones requeridas por la ley para otogar la indemización: existencia de lesiones gravísimas (interpretadas según los supuestos taxativamente previstos en el artículo 318 del Código Penal) y, en su caso, la existencia de un nexo de causalidad con el accionar de los agentes del Estado. Si bien la ley $\mathrm{N}^{\mathrm{o}} 18.596$ no remite expresamente al artículo 318 del Código Penal, la Comisión Especial tomó el criterio de remitirse a esa definición, la única presente en nuestro derecho positivo.

Desde hace una década el Departamento de Medicina Legal y Ciencias Forenses de la Facultad de Medicina ha colaborado honorariamente y por razones humanitarias con la Comisión Especial, lo que fue formalizado en el convenio suscrito el 19 de octubre de 2016 entre el Ministerio de Educación y Cultura y la Universidad de la República. El objeto del convenio es colaborar con los informes periciales que le sean requeridos, así como contribuir a la formación y el entrenamiento de profesionales jóvenes en la valoración y documentación de lesiones y secuelas de la tortura y otros tratos crueles, inhumanos o degradantes ${ }^{(7,10,11)}$.

El propósito de este reporte es sistemzatizar los informes elaborados en los diez primeros años de aplicación de la ley en lo que refiere a las solicitudes de amparo presentadas por personas que alegaron haber sufrido lesiones gravísimas a raíz, o en ocasión del accionar de agentes del Estado en el período comprendido entre 13 de junio de 1968 y 28 de febrero de 1985. 


\section{Objetivos}

\section{Objetivo general}

Contribuir al conocimiento público de la actuación ilegítima y el terrorismo de Estado en Uruguay en el período 13/6/1968-28/2/1985 y del aporte realizado por la Medicina Legal en materia de verdad, justicia, memoria, reparación y garantía de no repetición, en relación con esos hechos.

\section{Objetivos específicos}

1. Sistematizar los informes realizados a partir de las solicitudes de indemnización motivadas por la alegación de haber sufrido lesiones gravísimas a raíz o en ocasión del accionar de agentes del Estado.

2. Conocer la valoración médico-legal a que dieron lugar y la resolución final tomada por la Comisión Especial.

\section{Material y método}

Se estudiaron los expedientes de solicitudes de indemnización por lesiones gravísimas a raíz o en ocasión del accionar de agentes del Estado en el período durante los primeros diez años de vigencia de la ley $\mathrm{N}^{\circ} 18.596$ (18/9/2009-17/9/2019).

Se analizaron las siguientes variables:

a) Fecha de solicitud (día/mes/año).

b) Fecha de resolución de la solicitud (día/mes/año).

c) Circunstancia alegada de producción de las lesiones (prisión, tortura, represión en la vía pública, otra).

d) Naturaleza de las lesiones alegadas: físicas (especificando), psíquicas (especificando).

e) Edad al momento de producción de las lesiones alegadas (expresada en años cumplidos; cuando se alega que resultaron de una agresión prolongada en el tiempo, se tomó el momento de inicio).

f) Solicitud de informe médico-legal por la Comisión Especial (Sí/No; si lo hubo, institución responsable del informe: Ministerio de Salud o Universidad de la República (especificando: médico legista/psiquiatra/ambos).

g) Conclusiones del informe médico-legal sobre existencia de lesiones gravísimas (Sí/No); si las hubo, se indica la causal (según lo estipula el Código Penal): 1. Enfermedad cierta o probablemente incurable; 2. Pérdida de un sentido; 3 . Pérdida de un miembro o una mutilación que le torne inservible; 4. Pérdida de un órgano; 5. Pérdida de la capacidad de generar; 6. Grave y permanente dificultad de la palabra; 7. Deformación permanente del rostro; 8. Aborto.

h) Conclusiones del informe médico-legal sobre el nexo causal con la actuación de los agentes del Estado: 0 (sin nexo causal), 1 (no se afirma ni se descar- ta), 2 (no se asegura pero se estima probable), 3 (muy probable, razonable grado de certeza).

i) Tipo de lesiones que dieron lugar a las reparaciones: físicas, psíquicas, ambas (especificando).

j) Lapso transcurrido entre la producción de las lesiones gravísimas y la reparación (expresada en años cumplidos; cuando se alegó que las lesiones resultaron de una agresión prolongada en el tiempo, se tomó el momento de inicio).

Los resultados obtenidos se expresan en términos de frecuencias absolutas y relativas.

\section{Aspectos éticos}

La intervención del Departamento de Medicina Legal y Ciencias Forenses para la elaboración de los informes se produce a solicitud de la Comisón Especial de la ley $\mathrm{N}^{\mathrm{o}} 18.596$ y a partir de la presentación voluntaria de las personas que alegan haber sufrido lesiones gravísimas como consecuencia del accionar ilegítimo de los agentes del Estado.

El procesamiento de la información contenida en los expedientes se realizó con el conocimiento y aval expreso de: a) Comisión Especial creada al efecto por la ley $\mathrm{N}^{\circ}$ 18.596; b) Crysol, organización que nuclea a los exprisioneros políticos, a quienes representa en la Comisión Especial (ley No 18.596).

Los beneficios esperados para estas víctimas del terrorismo de Estado son parte de su reparación simbólica, a través del mejor conocimiento de los hechos del pasado reciente. Esto también constituye un beneficio para el conjunto de la sociedad. El conocimiento de estos hechos se enmarca en la política pública de verdad, justicia, memoria, reparación y garantía de no repetición.

Previo a la elaboración del reporte se elevó la consulta a la Comisión Nacional de Ética de la Investigación, la que la analizó y no encontró objeciones éticas a su elaboración y publicación (sesión de 10/3/2020).

\section{Resultados}

En el período 18/9/2009-17/9/2019 la Comisión Especial recibió 166 peticiones de personas que solicitaron ser indemnizadas al amparo del artículo 11, literal B de la ley $\mathrm{N}^{\mathrm{o}} 18.596$, alegando haber sufrido lesiones gravísimas a causa del accionar de los agentes del Estado.

De ellas, $115(\mathrm{FR}=0,70)$ fueron admitidas y 49 ( $F R=0,29)$ fueron desestimadas; en una de las solicitudes el solicitante falleció durante el trámite y en otra el interesado desistió de la petición. En las solicitudes desestimadas ( $\mathrm{n}=49$ ), a juicio de la Comisión Especial, los promotores no pudieron acreditar su condición de víctima $(n=5)$ o haber sufrido lesiones $(n=25)$, o bien las lesiones padecidas no fueron consideradas gravísimas o 
Tabla 1. Circunstancias en que se produjeron las lesiones gravísimas.

\begin{tabular}{lcl}
\hline Prisión política & 91 & 0,79 \\
Tortura & 82 & 0,71 \\
Represión en la vía pública & 9 & 0,08 \\
Violación & 11 & 0,10 \\
Exilio & 3 & 0,03 \\
Testigos de violencia & 2 & 0,02 \\
Durante la detención & 2 & 0,02 \\
$\begin{array}{l}\text { Otros (incluye enfrentamiento } \\
\text { armado, abandono y persecución } \\
\text { política) }\end{array}$ & 3 & 0,03 \\
\hline
\end{tabular}

no presentaban nexo causal con el accionar de los agentes del Estado $(\mathrm{n}=19)$.

Dentro de las solicitudes admitidas ( $\mathrm{n}=115), 74$ (FR: $0,64)$ correspondieron a hombres y $41(\mathrm{FR}: 0,36)$ a mujeres.

Las edades de las víctimas al momento de los hechos que produjeron el daño tuvo un rango de entre 1 y 47 años, con una media de 26 años. Se exceptúa un caso en que se admitió como lesión gravísima un daño producido en la etapa fetal.

La edad de las víctimas al momento de obtener la indemnización por lesiones gravísimas tuvo un rango de entre 38 y 86 años, con una media de 66 años.

El lapso transcurrido entre el momento en que se produjeron las lesiones gravísimas y la reparación fue de 39 años en promedio (rango: 28-49 años). El tiempo transcurrido entre el fin del período de terrorismo de Estado y la obtención del resarcimiento fue promedialmente de 28 años (rango: 25-34 años).

Las circunstancias alegadas en que se produjeron las lesiones gravísimas se presentan en la tabla 1 (en algunos casos se alegó más de una).

Todos los casos de violación alegada correspondieron a mujeres y los de abandono y testigos de violencia correspondieron a niños (todos menores de 13 años).

En la mayoría de los casos tramitados $(\mathrm{n}=109, \mathrm{FR}$ : 0,95) la Comisión Especial requirió informes complementarios a la documentación aportada por los solicitantes. Ellos fueron: informe médico-legal en 70 casos (FR: 0,61), informe psiquiátrico en 7 casos (FR: 0,06) e informes de ambas especialidades en 32 (FR: 0,28). En los restantes casos (n=6, FR: 0,05) la Comisión Especial se expidió en base a la información clínica aportada por la víctima.

El tipo de lesión gravísima, según la tipología establecida en el Código Penal, por la que se indemnizó a las
Tabla 2. Tipo de lesión gravísima indemnizada.

\begin{tabular}{lcc|}
\hline $\begin{array}{l}\text { Enfermedad cierta o } \\
\text { probablemente incurable }\end{array}$ & 102 & 0,89 \\
Aborto & 4 & 0,03 \\
$\begin{array}{l}\text { Deformación permanente del } \\
\text { rostro }\end{array}$ & 4 & 0,03 \\
$\begin{array}{l}\text { Pérdida de la capacidad para } \\
\text { generar }\end{array}$ & 3 & 0,03 \\
$\begin{array}{l}\text { Pérdida de un miembro o } \\
\text { mutilación que le tornó } \\
\text { inservible }\end{array}$ & 2 & 0,02 \\
$\begin{array}{l}\text { Grave y permanente dificultad } \\
\text { de la palabra, pérdida de un } \\
\text { órgano, pérdida de un sentido }\end{array}$ & 0 & \\
No especificada & 4 & 0,00 \\
\hline & & 0,03 \\
\hline
\end{tabular}

víctimas se detalla en la tabla 2 (en algunos casos fueron más de uno).

Las lesiones fueron de naturaleza física en 64 casos (FR: 0,56), psíquica en 43 casos (FR: 0,37) y mixta (FR: 0,05 ); en 2 casos no se obtuvieron datos (FR: 0,02). En las tablas 3 y 4 se detallan las lesiones físicas y psíquicas más frecuentes respectivamente.

Dentro de las alteraciones osteoarticulares predominaron ampliamente la artrosis $(\mathrm{n}=26)$ y las lesiones de columna $(n=16)$ y de hombro $(n=11)$.

Los trastornos por ansiedad correspondieron a trastorno por estrés postraumático crónico y los trastornos del humor a diferentes modalidades de depresión crónica. Los cuadros psicóticos incluyeron esquizofrenia, trastornos delirantes persecutorios y psicosis crónicas no especificadas.

La existencia de nexo causal entre las lesiones gravísimas constatadas con el accionar de los agentes del Estado fue estimado por los informes médico-legales o psiquiátricos, como se muestra en la tabla 5 .

\section{Discusión}

En los primeros diez años de vigencia de la ley $\mathrm{N}^{\circ}$ 18.596 se indemnizó a 115 personas por haber padecido lesiones gravísimas causadas por el accionar ilegítimo de los agentes del Estado. No se puede analizar esta cifra sin valorar las limitaciones que impuso la ley a esta causal indemnizatoria. Por una parte, el tipo penal de "lesiones gravísimas" es sumamente restrictivo, limitándolo a los casos de enfermedad incurable, la pérdida total de un miembro, un órgano o un sentido, el aborto, la pérdida total de la función reproductiva, la desfiguración permanente del rostro o la grave y permanente difi- 


\begin{tabular}{|lll|}
\hline $\begin{array}{l}\text { Tabla 3. Lesiones físicas gravísimas informadas con } \\
\text { mayor frecuencia. }\end{array}$ & 0,30 \\
\hline Osteoarticulares & 35 & 0,05 \\
Neurológicas & 6 & 0,05 \\
Cardio-circulatorio & 6 & 0,04 \\
Ginecológicas & 5 & 0,03 \\
Otológicas & 4 & 0,03 \\
Odontológicas & 3 & 0,03 \\
Nefrológicas & 3 & 0,03 \\
Gastrointestinales & 3 & 0,03 \\
Reproductivas & 3 & 0,02 \\
Alteraciones estéticas & 2 & 0,01 \\
Respiratorias & 1 & 0,01 \\
Endocrinológicas & 1 & 0,02 \\
Mal definidas & 2 & \\
\hline & & \\
\hline
\end{tabular}

cultad para la palabra. De este modo, quedan por fuera, por ejemplo, la mayoría de las secuelas sensoriales o las secuelas osteoarticulares que no configuren un proceso morboso activo (enfermedad) ${ }^{(11,12)}$.

Por otra parte, aun en los casos en que la lesión se corresponde con el tipo penal, la dificultad para establecer el nexo causal es considerable a partir del tiempo transcurrido (nótese que, según el período considerado por la ley, la fecha de su promulgación y el lapso incluido en este reporte, los lapsos posibles entre los hechos alegados y la solicitud indemnizatoria oscilan entre 24 y 41 años). A esto hay que agregar las dificultades para obtener documentación clínica que, de existir, estaba en poder de los propios agentes del Estado de la época y que un grupo de víctimas residió muchos años en el extranjero, entre otras dificultades.

Por ello, resulta razonable pensar que las restrictivas exigencias legales desalentaron las solicitudes que, aun así, resultaron desestimadas en un 30\% (sea por falta de documentación probatoria, porque los daños no se ajustaban al tipo de lesiones gravísimas o porque no se determinó razonablemente un nexo causal con el accionar de los agentes del Estado).

Respecto a la distribución por sexo (dos tercios de los indemnizados son varones), no es posible avanzar en el análisis de su significado, a falta de datos cuantitativos sistemáticos sobre cómo afectó la represión a hombres y mujeres, respectivamente, en el período contemplado en la ley.
Tabla 4. Lesiones psíquicas gravísimas informadas con mayor frecuencia.

\begin{tabular}{lcl}
\hline Trastornos por ansiedad & 22 & 0,19 \\
Trastornos del humor & 15 & 0,13 \\
Trastornos psicóticos & 15 & 0,13 \\
Trastornos de la & 4 & 0,03 \\
personalidad y los impulsos & & \\
Mal definidos & 5 & 0,04 \\
\hline
\end{tabular}

Tabla 5. Nexo de causalidad de las lesiones gravísimas indemnizadas con el accionar de los agentes del Estado, según los informes técnicos $(n=115)$.

\begin{tabular}{lcc}
\hline $\begin{array}{l}\text { Nivel } 0 \text { - No se puede } \\
\text { establecer nexo causal }\end{array}$ & 9 & 0,08 \\
$\begin{array}{l}\text { Nivel } 1 \text { - No se afirma ni se } \\
\text { descarta }\end{array}$ & 30 & 0,26 \\
$\begin{array}{l}\text { Nivel } 2 \text { - No se afirma pero } \\
\text { se estima probable }\end{array}$ & 19 & 0,16 \\
$\begin{array}{l}\text { Nivel } 3 \text { - Muy probable, } \\
\text { razonable grado de certeza }\end{array}$ & 55 & 0,48 \\
Sin datos & 2 & 0,02 \\
\hline
\end{tabular}

La edad promedio de la víctimas al momento de los hechos (26 años) da cuenta del perfil de las víctimas de la tortura y demás formas de accionar ilegítimo del Estado, aunque seguramente está muy influido por el largo tiempo transcurrido hasta el momento de la reparación (39 años en promedio), con una previsible mortalidad.

Resulta significativo que una vez terminado el período del terrorismo de Estado y ya recuperada la institucionalidad democrática plena, las reparaciones demoraron 28 años en promedio. Esto significó que las víctimas la recibieran a una edad bastante avanzada (66 años en promedio).

Los resultados del análisis de las circunstancias en que se produjeron las lesiones gravísimas muestran un gran predominio de la prisión política prolongada y la tortura, lo que coincide con el patrón represivo impuesto en Uruguay que describen los estudios históricos ${ }^{(13,14)}$.

Merece destacar el dato de que de las 41 mujeres indemnizadas, 11 refirieron la violación como la causa de sus lesiones gravísimas físicas o psíquicas, lo que también confirma el componente de género, ya conocido en el accionar de los agentes del Estado de la época ${ }^{(13)}$. En 
todo caso, cabe enfatizar que esa cifra no es la de la violencia sexual, sino únicamente de la porción de la violencia sexual que causó lesiones gravísimas que pudieron ser certificadas varias décadas más tarde.

El tipo y la naturaleza de las lesiones gravísimas indemnizadas se corresponden con la alta participación de víctimas de tortura en la población estudiada. Un estudio nacional anterior que detalla los métodos de tortura usados por el terrorismo de Estado en Uruguay y su eficacia para ocasionar lesiones graves y gravísimas, señaló que la posibilidad de producción de una enfermedad cierta o probablemente incurable involucraba a todos los métodos de tortura ${ }^{(11)}$. Esto se corresponde con los resultados de estos diez años estudiados $(89 \%$ de los casos indemnizados correspondió a esta causal). También en coincidencia con ese trabajo y otros previos, dentro de estas enfermedades tuvieron alta presencia las artrosis postraumáticas y, en particular, las patologías de hombro causadas por "el gancho" (suspensión palestina $)^{(11-14)}$. También se alertaba sobre la posibilidad de abortos en las mujeres embarazadas sometidas a torturas, lo que también se evidencia en nuestros resulta$\operatorname{dos}^{(11)}$.

En coincidencia con la bibliografía nacional y extranjera, en el grupo de víctimas indemnizadas por lesiones gravísimas se evidenció la existencia de un daño psíquico a largo plazo ${ }^{(11,15-17)}$. Su manifestación fue polimorfa, con un marcado predominio de las manifestaciones por ansiedad, típicamente trastorno por estrés postraumático crónico, y la depresión. En este reporte se destaca, además, una considerable y llamativa presencia de trastornos psicóticos derivados del padecimiento de la tortura.

Las dificultades para demostrar el nexo causal que surge de nuestros resultados son propias del trabajo médico-legal requerido para acreditar los exigentes extremos impuestos por la ley para este tipo de reparación. Es oportuno insistir en que resulta un imperativo ético que los informes periciales deben ser de naturaleza estrictamente técnica ${ }^{(18,19)}$. Por el contrario, la opción de flexibilizar los criterios exigidos en favor de las personas peticionantes puede ser una opción legal, jurídica o administrativa perfectamente válida.

La indemnización de este grupo de víctimas, que permitió ofrecerles alguna forma de reparación pecuniaria y simbólica, requirió informes psiquiátricos y médico-legales en los que tuvo importante participación la Facultad de Medicina de la Universidad de la República y, en particular, su Departamento de Medicina Legal y Ciencias Forenses. Se ratifica así el rol de la Acción Forense Humanitaria en la prosecución de los objetivos de verdad, justicia, memoria y reparación. Lo anterior evidencia y ratifica las características del modelo uruguayo de Acción Forense Humanitaria, sustentada en la institucionalidad pública, con una fuerte participación de la Universidad de la República ${ }^{(7)}$.

\section{Conclusiones}

1. En los primeros diez años de vigencia de la ley $\mathrm{N}^{\mathrm{o}}$ 18.596 se indemnizó a 115 personas por haber padecido lesiones gravísimas causadas por el accionar ilegítimo de los agentes del Estado.

2. Al momento de los hechos las víctimas tenían 26 años de edad y la reparación se resolvió 39 años más tarde (promedialmente).

3. La prisión política prolongada y la tortura fueron las principales circunstancias de producción de las lesiones gravísimas.

4. La violencia sexual tuvo un señalado rol en la producción de los daños comprobados en las víctimas mujeres.

5. Como era previsible, se encontró una alta participación de las patologías osteoarticulares, trastornos por estrés postraumático crónico y depresión. Además, se encontró una elevada cantidad de psicosis crónicas.

6. Los informes médico-legales y psiquiátricos, pese a las dificultades para establecer el nexo causal de los daños con el accionar de los agentes del Estado, fueron determinantes para demostrar la enorme mayoría de las lesiones gravísimas que fueron indemnizadas.

7. Estos resultados son expresión del modelo uruguayo de Acción Forense Humanitaria, caracterizado por asentar en la institucionalidad pública con una fuerte participación de la Universidad de la República.

\section{Summary}

The role of Legal Medicine in human rights, forensic science and humanitarian action is increasingly visible. Act 18,596 compensates those who prove to have experienced severe lesions by state agents.

Objective: to contribute to public awareness of the illegitimate actions and state terrorism in Uruguay (June 13, 1968 to February 28, 1985) and the input of Legal Medicine in terms of truth, justice, memory, reparation and guarantees of non-repetition.

Method: the compensation requests for severe lesions received during the first 10 years the law was in force were studied.

Results and discussion: 115 out of 166 requests were admitted. In $95 \%$ of cases, a medico-legal and or psychological report was requested. Despite it being hard to prove the causal link, a link with a reasonable degree of certainty was found in 55 cases. Average age of victims at the time the lesions were caused was 26 years old. Average age at the time of compensation was 66 
years old. Lesions proved match those referred to in the national and international bibliography. The study points out the finding of a considerable number of psychotic disorders.

Conclusions: 115 victims were compensated for severe lesions caused, in the wide majority of cases, by long imprisonment and torture. A high prevalence of osteoarticular diseases was observed, chronic post-traumatic stress disorders and depression, although a high number of chronic psychoses, too. Medico-legal reports were relevant to demonstrate severe lesions and the causal link with the action of state agents.

\section{Summary}

Decree 158/019 introduced several amendments to the national law on ethical issues in research involving human subjects. Given the fact that these amendments are included in a decree that keeps most of the text of Decree $379 / 08$, it may happen that at least some of them are not noticed by readers who are not directly involved in research involving human subjects.

Learning about the above mentioned amendments is important since it includes modifications in the operation of ethical committees on institutional research and the National Ethics Commission, and also significant affirmations in connection with concepts that have to do with the timely use of placebo, informed consent in epidemiological studies and requirements for research conducted from abroad.

\section{Resumo}

O papel da Medicina Legal nos direitos humanos e ação forense humanitária é cada vez mais visível. A Lei $\mathrm{N}^{\circ}$ 18.596 indeniza aquelas pessoas que acreditem ter sofrido lesões gravíssimas causadas pela ação de agentes do Estado.

Objetivo: contribuir para o conhecimento público da ação ilegítima e o terrorismo de Estado no Uruguai no período 13 de junho de 1968 a 28 de fevereiro de 1985 e da contribuição da Medicina Legal em termos de verdade, justiça, memoria, reparação e garantia de não repetição.

Material e método: foram estudados os pedidos de indenização por lesões gravíssimas recebidas durante os primeiros dez anos de vigência da lei.

Resultados e discussão: das 166 solicitações recebidas, foram incluídas 115 . Em 95\% dos casos o laudo médico-legal e/ou psiquiátrico foi exigido. Embora fosse difícil demonstrar a relação causal, em 55 casos foi estabelecida com um razoável grau de certeza. A idade média das vítimas no momento em que as lesões foram produzidas era de 26 anos. A idade média no momento da indenização era de 66 anos. As lesões demostradas correspondem às referidas na bibliografia nacional e estrangeira. Destaca-se o achado da presença considerável de transtornos psicóticos.

Conclusões: foram indenizadas 115 vítimas por lesões gravíssimas causadas na grande maioria por prisão política prolongada e tortura. Observou-se uma alta prevalência de patologias osteoarticulares, transtornos por estresse pós-traumático crônico e depressão, mas também um número alto de psicoses crônicas. Os laudos médico-legais foram relevantes para demonstrar as lesões gravíssimas e sua relação causal com a ação dos agentes do Estado.

\section{Bibliografía}

1. Cordner S, Tidball-Bonz M. Humanitarian forensic action-Its origins and future. Forensic Sci Int 2017; 279:65-71.

2. Stan, CA. Forensic standardizations in torture and death in custody investigations. Interv Med App Sci 2012; 4(2):210-6.

3. Thomsen JL, Helweg-Larsen K, Rasmussen OV. Amnesty International and the forensic sciences. Am J Forensic Med Pathol 1984; 5(4):305-11.

4. Thomsen JL, Voigt J. Forensic medicine and human rights. Forensic Sci Int 1988; 36(1-2):147-51.

5. Thomsen JL. The role of the pathologist in human rights abuses. J Clin Pathol 2000; 53(8):569-72.

6. Saukko P, Knigth B. Abuse of human rights: deaths in custody. In: Saukko P, Knight B. Knigth's forensic pathology. $4^{\text {th }}$ ed. Florida: CRC Press, 2016: 299-310.

7. Rodríguez H. HFA-Uruguay. Disponible en: https://medicinalegal.edu.uy/hfa-uruguay/ [Consulta: 10 setiembre 2017].

8. Comisión para la Paz. Informe Final. Uruguay, 2003. Disponible en: www.archivo.presidencia.gub.uy/noticias/archivo/2003/abril/Informe_final.doc [Consulta: 10 setiembre 2017].

9. Rodríguez H, Verdú F. La autopsia histórica: presentación del método y su aplicación al estudio de un hecho violento ocurrido en Uruguay en el año 1972. Rev Méd Urug 2003; 19(2):126-39.

10. Rodríguez H. Legal medicine and forensic science in Uruguay. In: Ubelaker D. ed. The global practice of forensic science. Wiley: Oxford, 2015:335-49.

11. Rodríguez H, Borches F, Bazán N, Gamero S, Lozano F, Roó F. Métodos de tortura del terrorismo de estado en Uruguay y valoración médico-legal de su idoneidad para causar lesiones graves o gravísimas. Rev Meid Urug 2019; 35(1):42-53.

12. Rodríguez H, Olivera J, Alves A, Mederos D. Delitos contra la personalidad física: traumatismo, lesiones y violencia doméstica. En: Rodríguez H, coord. Medicina Legal. Derecho Médico. Montevideo: Oficina del Libro-FEFMUR, 2017:209-24.

13. Rico A, coord. Investigación histórica sobre dictadura y terrorismo de estado en Uruguay (1973-1985). Montevideo: UdelaR, 2008. 
14. Yarzábal L. La tortura como enfermedad endémica en América Latina: sus características en Uruguay. Nueva Antropol 1985; 7 (28):75-92.

15. Organización de las Naciones Unidas. Oficina del Alto Comisionado de Derechos Humanos. Manual para la investigación y documentación eficaces de la tortura y otros tratos o penas crueles, inhumanos o degradantes (Protocolo de Estambul). Ginebra: ONU, 2004.

16. Madariaga C. Trauma psicosocial, trastorno de estrés postraumático y tortura. Santiago de Chile: Cintras, 2002.
17. Huminuik K. Special competencies for psychological assessment of torture survivors. Transcult Psychiatr 2017;54(2):239-59.

18. Guía de principios éticos de la Red Iberoamericana de instituciones de Medicina Legal y Ciencias Forenses. Rev Méd Urug 2018; 34(2):102-12.

19. Thomsen JL. Ethical considerations for forensic scientists participating in humanitarian action: a personal reflection. Forensic Sci Int 2017:379-80.

\section{Declaración de confictos de interés}

Victoria Iglesias y Hugo Rodríguez Almada participaron en la elaboración de informes médico-legales a solicitud de la Comisión Especial de la ley $\mathrm{N}^{\circ} 18.596$.

Valeria da Trindade fue asesora letrada de la Comisión Especial creada de la ley $\mathrm{N}^{\mathrm{o}} 18.596$.

\section{Contribución de los autores}

Victoria Iglesias, https://orcid.org/0000-0003-1439-5608. Diseño, ejecución y redacción del artículo. Hugo Rodríguez Almada, https://orcid.org/0000-0002-7330-6508. Diseño, ejecución y redacción del artículo. Valeria da Trindade, https://orcid.org/0000-0003-4781-5955. Recolección del material, análisis del marco jurídico y redacción del artículo. 